\title{
Fatty Acid Composition and Arachidonate Metabolites in the Livers of Ethanol-Treated Rats Fed an Arachidonate-Supplemented Diet: Effect of Dietary Fat
}

\author{
Misako Okita, ${ }^{1, *}$ Takayo Sasagawa, ${ }^{1}$ Kazuhiko Suzuki, ${ }^{1}$ \\ Ayumu Miyamoto, ${ }^{2}$ Hiroyuki Wakabayashi, ${ }^{2}$ \\ and Akiharu WATANABE ${ }^{2}$ \\ ${ }^{1}$ Department of Nutritional Science, \\ Faculty of Health and Welfare Science, \\ Okayama Prefectural University, Soja 719-1197, Japan \\ ${ }^{2}$ The 3rd Department of Internal Medicine, \\ Toyama Medical and Pharmaceutical University, \\ Toyama 930-0194, Japan
}

(Received December 5, 1997)

Summary The effects of $1 \%$ arachidonic acid ethyl ester (AA) administration on the liver prostanoid metabolites and on serum and liver lipids in $3 \mathrm{~g} / \mathrm{kg}$ ethanol-administered rats fed $10 \%$ lard or corn oil were studied. The rats were divided into 6 groups: lard-sucrose (Lard-Suc); lard-ethanol without AA (Lard-Et); lard-ethanol with AA (Lard-EtAA); corn oil-sucrose (Corn-Suc); corn oil-ethanol without AA (Corn-Et); and corn oil-ethanol with AA (Corn-EtAA). Liver triglyceride increased in Corn-EtAA compared with Corn-Et. Arachidonic acid $(20: 4 n-6)$ levels in liver phospholipid were significantly decreased in Corn-Et, but elevated in Lard-Et. The levels of 20:4n-6 were significantly increased with AA administration in both ethanol groups. Liver 6-keto-prostaglandin F1 $\alpha$ (6-keto-PGF1 $\alpha)$ in Corn-Suc $(24.7 \pm 5.1 \mathrm{pg} / \mathrm{mg}$ protein) was markedly higher than in Lard-Suc $(4.5 \pm 1.2 \mathrm{pg} / \mathrm{mg}$ protein), and the 6-keto-PGF $1 \alpha$ lowered significantly with the addition of ethanol $(9.3 \pm 0.9 \mathrm{pg} / \mathrm{mg}$ protein), but it increased with AA administration $(21.6 \pm 4.9 \mathrm{pg} / \mathrm{mg}$ protein). In Lard-EtAA, a significant increase in 6-keto-PGF1 $\alpha$ was observed compared with Lard-Suc. The liver leukotriene $\mathrm{B}_{4}\left(\mathrm{LTB}_{4}\right)$ level in Lard-Suc was significantly lower than that of Corn-Suc. In the corn oil group, ethanol feeding was associated with a significant increase in liver $\mathrm{LTB}_{4}$. AA administration to Corn-Et suppressed the elevated LTB $_{4}$. Serum thiobarbituric acid-reactive substance (TBARS) concentrations in the corn oil group were higher than in the lard group, and these concentrations were not altered by AA administration. From these results, we concluded

* To whom correspondence should be addressed. 
that the administration of AA in rats treated with ethanol increased $20: 4 n-6$ in liver phospholipid and liver $\mathrm{PGI}_{2}$ levels, irrespective of dietary fat, and may protect against alcoholic liver injury. AA with a diet rich in linoleic acid $(18: 2 n-6)$, however, may increase fat in the alcoholic liver.

Key Words ethanol fed rats, arachidonic acid ethyl ester, dietary fat, 6-keto-prostaglandin $\mathrm{F} 1 \alpha$, leukotriene $\mathrm{B}_{4}$

The amounts and fatty acid composition of dietary fat have been reported to have an important role in alcoholic liver injury $(1,2)$. A common finding in ethanol-treated animals is a change in the fatty acid composition of phospholipids, the increased levels of oleic acid (18:1) and linoleic acid $(18: 2 n-6)$, and the decreased level of arachidonic acid $(20: 4 n-6)(3)$. It has been suggested that the increase in $18: 2 n-6$ and the decrease in $20: 4 n-6$ induced by ethanol are caused by the reduction in $\Delta 6$ and $\Delta 5$ desaturase activity $(4,5)$. Since the severity of liver pathology correlated negatively with the decrease in 20:4n-6 in ethanolfed rats, the increased metabolism of $20: 4 n-6$ into epoxides, such as 14,15-epoxyeicosatrienoic acid (EET), 11,12-EET, and 8,9-EET, and the decrease in 20:4n-6 synthesis were proposed as important factors in ethanol-induced liver injury (6). As a mechanism of alcoholic liver injury, it was also supposed that inhibited metabolism of $18: 2 n-6$ into $20: 4 n-6$ induced an increase in the generation of free radicals by the action of lipoxygenase on $18: 2 n-6$ (2).

Arachidonic acid is especially important for membrane function and for the formation of prostanoids. In the liver, nonparenchymal cells, such as sinusoidal endothelial cells (7) and Kupffer cells (8), are known to produce significant amounts of prostaglandin (PG) $\mathrm{I}_{2}$ and $\mathrm{PGE}_{2}$. Kupffer cells have been known to produce leukotrienes (LTs) in response to various stimuli (8). Rat hepatocyte, however, produced only a small amount of $\mathrm{PGI}_{2}(9)$. $\mathrm{PGI}_{2}$ is especially protective against ischemia-induced hepatic injury (10). Although the protective effect of PG on alcoholic liver injury is unknown, the severity of liver injury, i.e., fatty liver, necrosis, and inflammation in experimental rats fed ethanol, correlated negatively with plasma $\mathrm{PGE}_{2}$ and positively with plasma $\mathrm{LTB}_{4}(11)$.

In a previous study (12), we recognized a decrease in triglyceride and an increase in 6-keto-PGF1 $\alpha$, a stable metabolite of $\mathrm{PGI}_{2}$, in the livers of ethanol-treated rats fed a lard diet supplemented with arachidonic acid ethyl ester (AA). The aim of the present study was to clarify the effect of the dietary fat on the liver and serum triglyceride, liver fatty acid, and liver arachidonate metabolites in ethanol-treated rats fed an AA-supplemented diet.

\section{MATERIALS AND METHODS}

Animals and diets. Eight-week-old male Sprague-Dawley rats (Charles River Japan, Tokyo), 8 weeks old, weighing 250-300 g each, were used. The rats were 
Table 1. Fatty-acid composition of dietary fats.

\begin{tabular}{|c|c|c|c|c|}
\hline \multirow[b]{2}{*}{$\begin{array}{l}\text { Arachidonic acid- } \\
\text { ethyl ester }(\%)\end{array}$} & \multicolumn{2}{|c|}{ Lard } & \multicolumn{2}{|c|}{ Corn oil } \\
\hline & 0 & 10 & 0 & 10 \\
\hline \multicolumn{5}{|c|}{ Fatty acid (weight \%) } \\
\hline $14: 0$ & 2.0 & 1.8 & - & - \\
\hline $16: 0$ & 26.5 & 23.9 & 11.2 & 10.1 \\
\hline $16: 1 n-7$ & 3.7 & 3.5 & - & - \\
\hline $18: 0$ & 12.1 & 10.9 & 2.1 & 1.9 \\
\hline $18: 1 n-9$ & 42.5 & 38.3 & 34.7 & 31.4 \\
\hline $18: 2 n-6$ & 9.8 & 8.8 & 50.5 & 45.7 \\
\hline $18: 3 n-3$ & 0.7 & 0.6 & 1.5 & 1.4 \\
\hline $20: 3 n-6$ & - & 0.2 & - & 0.2 \\
\hline $20: 4 n-6$ & 0.3 & 9.6 & - & 9.3 \\
\hline Total $n-6$ & 10.1 & 18.6 & 50.5 & 55.2 \\
\hline
\end{tabular}

housed separately and provided with standard laboratory chow (type MF, Oriental Yeast, Tokyo) and water ad libitum for more than a week before the start of the experimental feeding.

Two kinds of dietary fat, lard and corn oil, containing 9.8\% and 50.5\% linoleic acid, respectively, were used to compare the effects of dietary $n-6$ fatty acid intake with the administration of AA. The purified diet consisted of $25 \%$ casein (by weight), $10 \% \alpha$-starch, $37.5 \%$ cornstarch, $5 \%$ sucrose, $8 \%$ cellulose, $3.5 \%$ mineral mix (AIN formulation, Oriental Yeast), 1\% vitamin mix (modified AIN formulation containing 20\% choline bitartrate, Oriental Yeast), 10\% fat (corn oil or lard), and $0.02 \% \delta$-tocopherol. AA ( $10 \%$ fat content, w/w), donated by Suntory, Tokyo, was added. $\delta$-Tocopherol was mixed to the diets to prevent autooxidation of AA. Fatty acid compositions of the dietary fats are summarized in Table 1.

A single daily dose of $3 \mathrm{~g} / \mathrm{kg}$ body weight of ethanol was given intragastrically between noon and 1 p.m. for 14 d. Control rats were given isocaloric sucrose instead of ethanol.

The rats were randomly allocated to 6 groups, with 6 rats in each group: lard-sucrose (Lard-Suc); lard-ethanol without AA (Lard-Et); lard-ethanol with AA (Lard-EtAA); corn oil-sucrose (Corn-Suc); corn oil-ethanol without AA (Corn-Et); and corn oil-ethanol with AA (Corn-EtAA). The rats in the sucrose and ethanol groups were pair-fed for $14 \mathrm{~h}$ ( 7 p.m. to 9 a.m.) and kept under standard conditions: a temperature of $24 \pm 2{ }^{\circ} \mathrm{C}$; darkness from 7 p.m. to 7 a.m.; and an adequate water supply. The AA-supplemented diets were prepared every day immediately before feeding to avoid autooxidation of AA.

After the 14-d experimental period, the rats were fasted overnight for an analysis of serum triglyceride level and were then anesthetized with diethyl ether. Blood was collected by cardiac puncture into polystyrene tubes. The liver was removed 
immediately after blood sampling, washed with ice-cold saline, blotted, and weighed. Blood samples were centrifuged at $1,630 \times g$ for $15 \mathrm{~min}$ at $4^{\circ} \mathrm{C}$ to obtain serum, and the sera were stored at $-30^{\circ} \mathrm{C}$.

Blood analysis. Serum alanine aminotransferase (ALT) activity was measured by the ultraviolet method, using an autoanalyzer (Hitachi 7170, Hitachi, Tokyo).

Lipid analysis. Triglyceride levels were determined by using triglyceride E enzymatic kits (Wako Pure Chemicals, Osaka). Total lipids in the livers were extracted by the Folch method (13). The lipids were separated into phospholipid, triglyceride, and cholesterol ester fractions by thin-layer chromatography by using a solvent system of petroleum ether/ethyl ether/acetic acid $(80: 20: 1$, by vol). Butylated hydroxytoluene was added as an antioxidant at a concentration of $0.005 \%$ $(\mathrm{w} / \mathrm{v})$ to the solvents used for lipid extraction. Fatty acid composition in phospholipid was analyzed according to a method described previously (14).

As an index of lipid peroxidation, thiobarbituric acid-reactive substances (TBARS) were measured according to the method described by Yagi (15).

Measurement of 6-keto-PGFl $\alpha$ and LTB $_{4}$. About $100 \mathrm{mg}$ of liver tissue was rinsed with chilled saline containing $0.1 \mathrm{~mm}$ indomethacin and $10 \mathrm{~mm}$ EDTA immediately after it was obtained. An analysis of the stable $\mathrm{PGI}_{2}$ hydrolysis product, 6-keto-PGF $1 \alpha$ and $\mathrm{LTB}_{4}$, was carried out according to the method of Kawada et al (16). Protein content was estimated by the Lowry method (17).

Statistical analysis. Results are expressed as the mean \pm SE. The statistical analysis was performed on a PC using commercially available software (STATISTICA/Mac, Three's Company, Tulsa, Oklahoma, USA). Two-way ANOVA was performed to determine correlations among the treatments and diets, and at the detection of a significant effect, the differences between groups were determined by Duncan's multiple-range test. The correlation coefficient was calculated by the Pearson test. $p$ values were considered significant at less than 0.05 .

\section{RESULTS}

\section{Relative liver weight, triglyceride content, and serum ALT activity}

Significant interactions between treatment and diet were observed in the serum ALT activity and the liver triglyceride content (Table 2).

Serum ALT activity was higher in the Lard-Et group than in the Lard-Suc group. In the Corn-Et group, although the serum ALT activity was not elevated compared with the Corn-Suc group, the administration of AA induced a significantly higher ALT activity compared with that in the Corn-Suc group. The serum ALT activity correlated positively with liver triglyceride content $(r=0.419, p<0.05)$.

The liver triglyceride increased significantly in the Corn-EtAA group, but not in the Lard-EtAA group compared with the ethanol administered group in each diet. Serum triglyceride was increased in the Corn-Et group compared with the Corn-Suc group and was decreased significantly in the Corn-EtAA group compared with the Corn-Et group. In lard-fed groups, the triglyceride levels in liver and in 
Arachidonate Supplement in Ethanol-Treated Rats

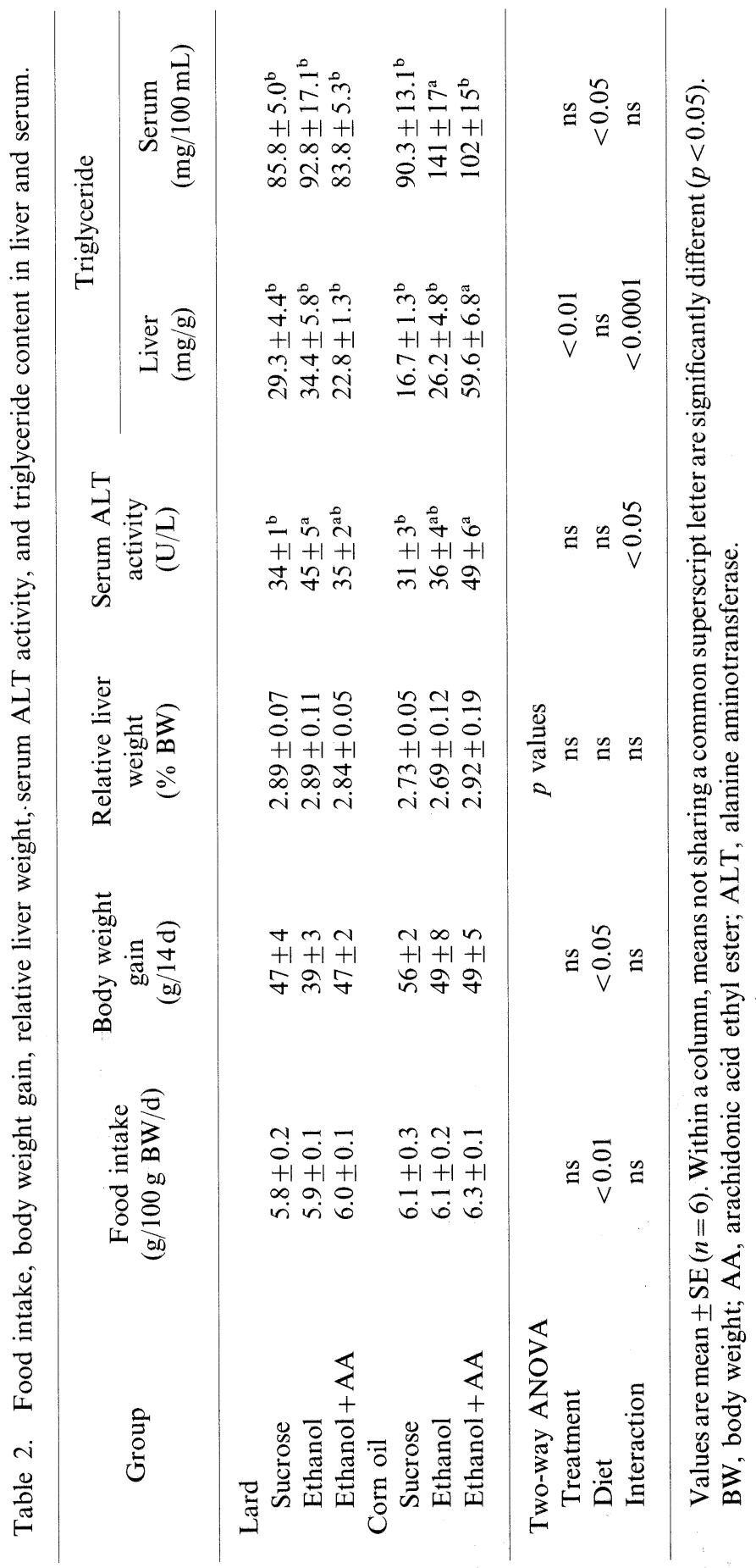


serum showed no significant alterations after the administration of ethanol and AA.

Although diet had some effect on food intake and body weight gain, there were no significant differences in food intake, body weight gain, or relative liver weight among the groups.

\section{Fatty acid composition in liver phospholipid}

Fatty acid composition of liver phospholipid is summarized in Table 3. The treatment and diet had significant effects on the fatty acid composition of liver phospholipid. In the Lard-Suc group, saturated fatty acid accounted for about $50 \%$ of fatty acid in the liver phospholipids. Linoleic acid and 20:4n-6 were plentiful in the Corn-Suc group. Increased $18: 1,18: 2 n-6$, and $20: 4 n-6$ and decreased palmitic acid $(16: 0)$ were observed in the Lard-Et group compared with the Lard-Suc group. In the Corn-Et group, $20: 4 n-6$ and $20: 4 n-6 / 18: 2 n-6$ were lower and $18: 1$ was higher than those in the Corn-Suc group. A marked elevation in $20: 4 n-6$ and $20: 4 n-6 / 18: 2 n-6$ levels and a subsequent significant decrease in $18: 1$ and $18: 2 n-6$ were seen in the Lard-EtAA group compared with the Lard-Et group. The Corn-EtAA group also exhibited increased levels of $20: 4 n-6$ and $20: 4 n-6 / 18: 2 n-6$ compared with the Corn-Et group.

\section{Liver prostaglandin and leukotriene content}

The contents of 6-keto-PGF1 $\alpha$, the major metabolite of $\mathrm{PGI}_{2}$, and of $\mathrm{LTB}_{4}$ in the liver are shown in Fig. 1. The significant effects of treatment and diet on liver 6-keto-PGF1 $\alpha(p<0.001)$ and on $\mathrm{LTB}_{4}(p<0.05)$ levels were observed. The 6-keto-PGF1 $\alpha$ level in the Corn-Suc group $(24.7 \pm 5.1 \mathrm{pg} / \mathrm{mg}$ protein) was markedly higher than in the Lard-Suc group $(4.5 \pm 1.2 \mathrm{pg} / \mathrm{mg}$ protein). The 6-keto-PGF1 $\alpha$ level in the Corn-Suc group was significantly lowered in the Corn-Et group $(9.3 \pm 0.9 \mathrm{pg} / \mathrm{mg}$ protein $)$ and increased in the Corn-EtAA group $(21.6 \pm 4.9 \mathrm{pg} / \mathrm{mg}$ protein). In lard-fed rats, however, the 6-keto-PGF1 $\alpha$ level showed no significant changes after ethanol feeding compared with sucrose feeding, though the AAadministered group showed a significantly higher level compared with the LardSuc group.

The $\mathrm{LTB}_{4}$ level in the Lard-Suc group $(4.0 \pm 0.3 \mathrm{pg} / \mathrm{mg}$ protein $)$ was significantly lower than in the Corn-Suc group $(7.4 \pm 0.3 \mathrm{pg} / \mathrm{mg}$ protein). Ethanol feeding was associated with significantly higher $\mathrm{LTB}_{4}$ levels in the corn-oil group than in the sucrose group. The administration of AA to the Corn-Et group induced some decrease in $\mathrm{LTB}_{4}$ level, but no significant differences were observed between the $\mathrm{LTB}_{4}$ levels in the Corn-Suc and Corn-EtAA groups. In the Lard-EtAA group, however, $\mathrm{LTB}_{4}$ levels were increased compared with the Lard-Suc group.

Serum TBARS contents

Dietary fat had a significant effect (two-way ANOVA, $p<0.05$ ) on serum TBARS content (Fig. 2). The TBARS content in the Lard-Et group (4.3 \pm 


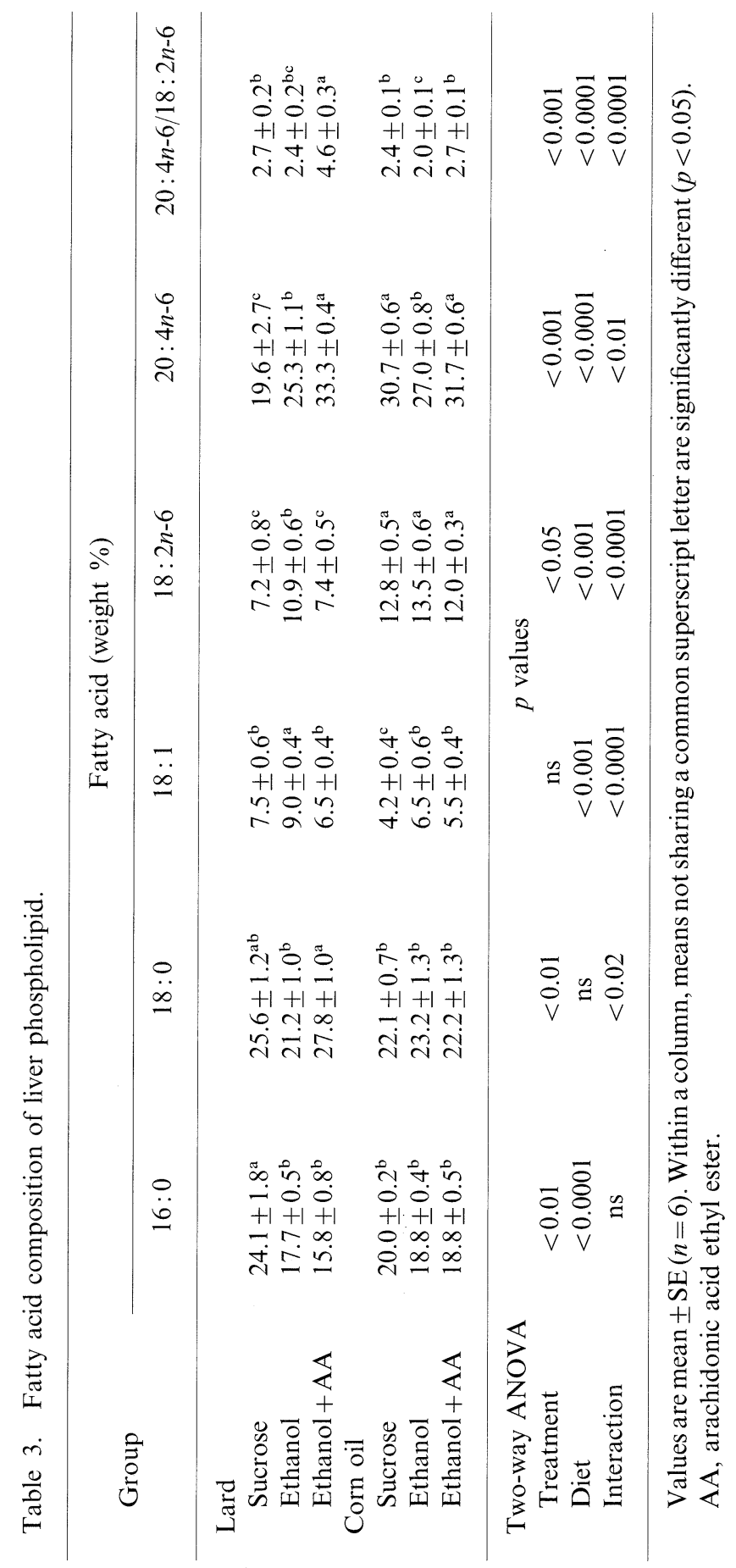




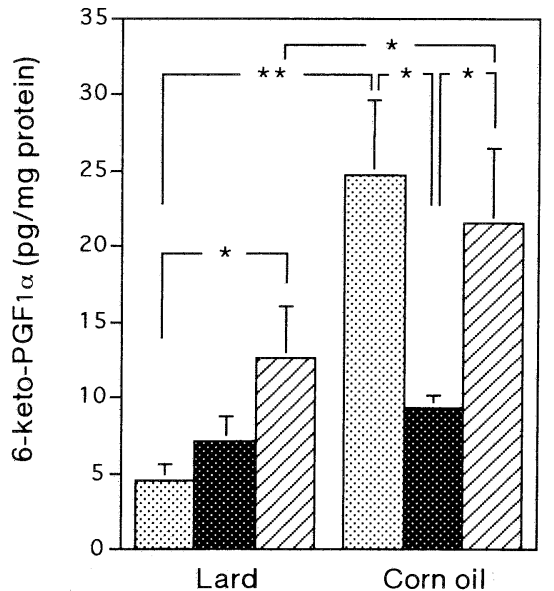

Sucrose

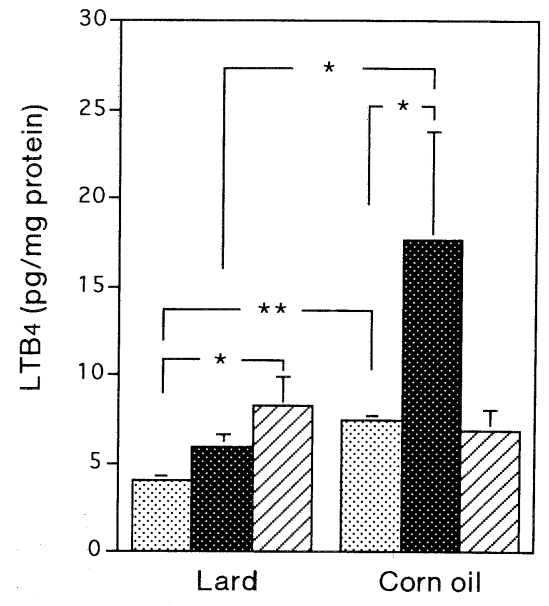

$\mathbb{Z}$ Ethanol $+A A$

Fig. 1. Liver 6-keto-PGF1 $\alpha$ ( $n=6$ in each group) and $\mathrm{LTB}_{4}$ (in corn oil-fed rats, $n=3$ in each group; in lard-fed rats, $n=6$ in each group) content in lard- and corn oil-fed rats with or without ethanol feeding and AA administration. ${ }^{* *} p<0.01,{ }^{*} p<0.05$.

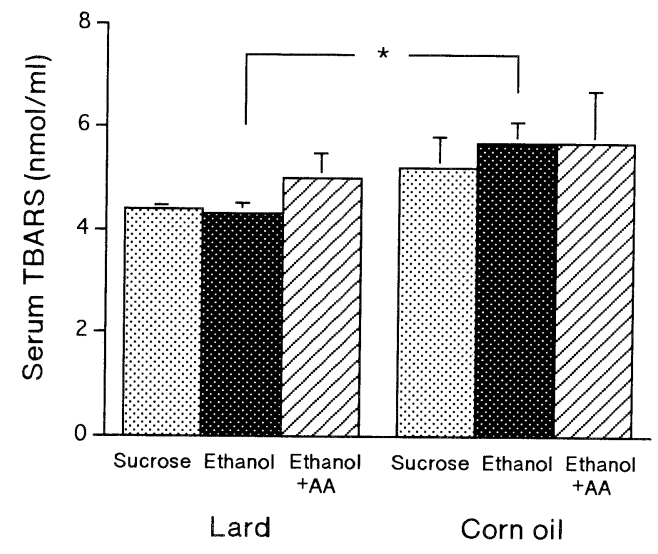

Fig. 2. Serum thiobarbituric acid-reactive substances (TBARS) in lard- and corn oil-fed rats with or without ethanol and AA administration ( $n=6$ in each group). ${ }^{*} p<$ 0.05 .

$0.2 \mathrm{nmol} / \mathrm{mL})$ was significantly lower than in the Corn-Et group $(5.7 \pm 0.4 \mathrm{nmol} / \mathrm{mL})$. The administration of AA to the ethanol-fed group did not change the serum TBARS level in either dietary fat group. 


\section{DISCUSSION}

Dietary lard and corn oil differently affected the consequences of ethanol feeding and of AA administration in the present study. The lard and corn oil diets contained 2.2 and 11.4 energy $\%$ of $18: 2 n-6$, respectively, and the rats fasted overnight. Fremont and Gozzelino (18) reported that in overnight-fasted rats fed two different diets containing low (3.0 energy \%) and high ( 8.9 energy \%) $18: 2 n-6$, the $18: 2 n-6$ and $20: 4 n-6$ levels in the liver phospholipid were 6.8 and $33.9 \mathrm{~mol} \%$ (low $18: 2 n-6$ diet) and 9.0 and $34.2 \mathrm{~mol} \%$ (high $18: 2 n-6$ diet), respectively. Only 19.6 weight $\%$ was shown by $20: 4 n-6$ in the liver phospholipid in our Lard-Suc group. A lower $18: 2 n-6$ energy $\%$ in our lard-fed group may explain the lower $20: 4 n-6$ level.

Hepatic $\Delta 6$ desaturase activity has been shown to decrease after longtime (24-96h) food deprivation (19-2l), but the activity was not modified in short-term $(15 \mathrm{~h})$ fasting $(21)$. In the present study, therefore, desaturation of $18: 2 n-6$ may be slightly affected by overnight fast. The low $20: 4 n-6 / 18: 2 n-6$ ratios observed in corn oil-fed rats could be attributed to their high $18: 2 n-6$ levels, because $\Delta 6$ desaturase activity has been decreased by dietary $18: 2 n-6$ enrichment $(22,23)$ and was suggested to be enhanced by the decreased availability of $18: 2 n-6$ for substrate (24).

After a 7-d exposure to alcohol, a $44 \%$ loss of $20: 4 n-6$ was induced in rat liver fed with a low $18: 2 n-6(1.3$ energy \%) diet (25). In another report (26), a 55\% loss of $20: 4 n-6$ and a $58 \%$ increase of $18: 2 n-6$ were observed in rats fed alcohol and corn oil ( $50 \%$ energy). In the present study, ethanol feeding induced a significant decrease of $20: 4 n-6$ in rats fed corn oil, but not in rats fed lard. This result in lard-fed rats is supported by a previous report of ours (12) and another report (27) showing an increased 20:4n-6 level in liver microsome obtained from rats fed saturated fat and ethanol.

An increase in 20:4n-6 with ethanol administration in lard-fed rats may be one possible mechanism for reducing fat accumulation in rat liver, as proposed by Nanji et al (27). A suppression of fatty acid synthase gene expression by albumin-bound 20:4n-6 was observed in primary cultures of rat liver cells (28). Although it was not statistically significant, the liver triglyceride levels in the Lard-Suc group were higher than those in the Corn-Suc group, having a high $20: 4 n-6$ level in the liver phospholipid. However, the significantly high level of liver triglyceride in the Corn-EtAA group cannot be explained by the liver's $20: 4 n$ 6 level. Fatty-acid oxidation or very low density lipoprotein secretion may be affected by the administration of ethanol and AA in a high $18: 2 n-6$ diet.

Mann et al (29) observed that dietary AA led to increases in plasma and aorta phospholipid $20: 4 n-6$ in rats, and that these changes were associated with an increase in $\mathrm{PGI}_{2}$ metabolites excreted in the urine. We also found that liver 6-keto-PGF1 $\alpha$ was significantly increased in the Corn-Suc group, having high $18: 2 n-6$ and $20: 4 n-6$ levels in liver phospholipid, and was markedly reduced by ethanol feeding. However, no reductions were found in liver 6-keto-PGF1 $\alpha$ with ethanol in lard-fed rats. To date, no evidence suggests that ethanol impairs PG 
synthesis; thus these results may be explained by the finding that PG synthesis is regulated by the availability of $20: 4 n-6(8)$. Our results also suggest that $20: 4 n-6$ levels in liver phospholipid may regulate PG synthesis in the liver, since liver 6-keto-PGF1 $\alpha$ content is changed parallel $(r=0.405, p<0.05)$ to the liver phospholipid 20:4n-6. Moreover, the low level of liver 6-keto-PGF1 $\alpha$ in the Lard-Suc group may be induced by down-regulation of cyclooxygenase-2, which was observed in the rats fed a diet rich in saturated fatty acid, as reported by Nanji et al (30).

$\mathrm{LTB}_{4}$, which is thought to be an extremely important mediator of inflammatory infiltration, enhances the activity of natural cytotoxic $\mathrm{T}$ cells. A cytotoxic $\mathrm{T}$ lymphocyte-hepatocyte interaction has been suggested to play a role, perhaps via cytokine production, in the pathogenesis of alcoholic liver disease (31). From our results, it appears that $\mathrm{LTB}_{4}$ production in the liver, which is accelerated by ethanol, is not affected by the administration of AA. In the corn oil-fed rats, liver $\mathrm{LTB}_{4}$ levels contrasted with the level of 6-keto-PGF1 $\alpha$. Because the administration of $\mathrm{PGI}_{2}$ protected against ischemia-induced hepatic injury (10) and $\mathrm{CCl}_{4}$-induced cell damage and death in rats (32), a reduction of lipid peroxidation has been proposed as an explanation for the hepatoprotective action of $\mathrm{PGI}_{2}$. A decrease in $\mathrm{PGI}_{2}$ with ethanol in corn oil-fed rats may increase lipid peroxidation (Fig. 2) and $\mathrm{LTB}_{4}$ production.

Dietary fat had a different effect on the serum TBARS in this study. It was shown that ethanol regimens low in vitamin E increased the hepatic TBARS (33). An intake of vitamin $\mathrm{E}$ higher in lard-fed rats $(4.63 \mathrm{mg} \alpha$-tocopherol/g PUFA: polyunsaturated fatty acid) than in corn oil-fed rats $(0.88 \mathrm{mg} \alpha$-tocopherol/g PUFA) in the present study may explain the lower TBARS level observed in the ethanol-treated rats fed lard.

We conclude that AA administration to rats treated with ethanol increases $\mathrm{PGI}_{2}$ level in the liver and that an elevated $\mathrm{PGI}_{2}$ level may protect against alcoholic liver injury, especially in rats fed a low $18: 2 n-6$ diet. An $18: 2 n$-6-rich diet with AA administration, however, may increase fat in an alcoholic liver.

This research was supported by Grants-in-Aid for Scientific Research from the Ministry of Education, Science, Sports and Culture of Japan.

\section{REFERENCES}

1) Nanji AA, French SW. 1986. Dietary factors and alcoholic cirrhosis. Alcohol Clin Exp Res 10: 271-273.

2) Nanji AA, French SW. 1989. Dietary linoleic acid is required for development of experimentally induced alcoholic liver injury. Life Sci 44: 223-227.

3) Reitz RC. 1993. Dietary fatty acids and alcohol: effects on cellular membranes. Alcohol Alcoholism 28: 59-71.

4) Nervi AM, Peluffo RO, Brenner RR, Leikin AI. 1980. Effect of ethanol administration on fatty acid desaturation. Lipids 15: 263-268. 
5) Nakamura MT, Tang AB, Villanueva J, Halsted CH, Phinney SD. 1994. Selective reduction of $\Delta 6$ and $\Delta 5$ desaturase activities but not $\Delta 9$ desaturase in micropigs chronically fed ethanol. $J$ Clin Invest 93: 450-454.

6) French SW, Morimoto M, Reitz RC, Koop D, Klopfenstein B, Estes K, Clot P, Ingelman-Sundberg M, Albano E. 1997. Lipid peroxidation, CYP2E1 and arachidonic acid metabolism in alcoholic liver disease in rats. $J$ Nutr 127: 907S-911S.

7) Rieder H, Ramadori G, Allmann K-H, Meyer zum Buschenfelde K-H. 1990. Prostanoid release of cultured liver sinusoidal endothelial cells in response to endotoxin and tumor necrosis factor. Comparison with umbilical vein endothelial cells. J Hepatol 11: 359-366.

8) Decker K. 1985. Eicosanoids, signal molecules of liver cells. Semin Liver Dis 5: 175-190.

9) Johnston DE, Peterson MB, Mion F, Berninger RW, Jefferson DM. 1991. Synthesis and degradation of eicosanoids in primary rat hepatocyte cultures. Prostaglandins Leukot Essent Fatty Acids 43: 119-132.

10) Sikujara O, Monden M, Toyoshima K, Okamura J, Kosaki G. 1983. Cytoprotective effect of prostaglandin $\mathrm{I}_{2}$ on ischemia-induced hepatic cell injury. Transplantation 36: 238-242.

11) Nanji AA, Khettry U, Sadrzadeh SMH, Yamanaka T. 1993. Severity of liver injury in experimental alcoholic liver disease. Correlation with plasma endotoxin, prostaglandin $\mathrm{E}_{2}$, leukotriene $\mathrm{B}_{4}$, and thromboxane $\mathrm{B}_{2}$. Am J Pathol 142: 367-373.

12) Okita M, Suzuki K, Sasagawa T, Yamamoto J, Miyamoto A, Wakabayashi H, Watanabe A. 1997. Effect of arachidonate on lipid metabolism in ethanol-treated rats fed with lard. J Nutr Sci Vitaminol 43: 311-326.

13) Folch J, Lees M, Sloane-Stanley GH. 1957. A simple method for the isolation and purification of total lipids from animal tissues. $J$ Biol Chem 226: 497-509.

14) Okita M, Yoshida S, Yamamoto J, Suzuki K, Kaneyuki T, Kubota M. 1995. n-3 and $n-6$ fatty acid intake and serum phospholipid fatty acid composition in middle-aged women living in rural and urban areas in Okayama Prefecture. J Nutr Sci Vitaminol 41: 313-323.

15) Yagi K. 1984. A simple fluorometric assay for lipoperoxide in blood plasma. In: Methods in Enzymology (Packer L, ed), Vol 105, p 328-331. Academic Press, Orlando, FL.

16) Kawada N, Mizoguchi Y, Kobayashi K, Yamamoto S, Morisawa S. 1990. Arachidonic acid metabolites in carbon tetrachloride-induced liver injury. Gastroenterol Jpn 25: 363-368.

17) Lowry OH, Roseburge NJ, Farr AL, Randall RT. 1951. Protein measurement with folin phenol reagent. $J$ Biol Chem 193: 265-275.

18) Fremont L, Gozzelino M-T. 1996. Dietary sunflower oil reduces plasma and liver triacylglycerols in fasting rats and is associated with decreased liver microsomal phosphatidate phosphohydrolase activity. Lipids 31: 871-878.

19) Peluffo RO, de Gomez Dumm NT, de Alaniz MJT, Brenner RR. 1971. Effect of protein and insulin on linoleic acid desaturation of normal and diabetic rats. J Nutr 101: 1075-1084.

20) Nervi AM, Catala A, Brenner RR, Peluffo RO. 1974. Dietary and hormonal effects upon activity of "soluble" protein and particulate fraction of fatty acid desaturation system of rat liver microsomes. Lipids 10: 348-352.

21) de Gomez INT, de Alaniz MJT, Brenner RR. 1976. Comparative effect of glucagon, dibutyryl cyclic AMP, and epinephrine on the desaturation and elongation of linoleic acid by rat liver microsomes. Lipids 11: 833-836.

22) Brenner RR. 1981. Nutritional and hormonal factors influencing desaturation of essential fatty acids. Prog Lipid Res 20: 41-47. 
23) Brenner RR, Garda H, de Gomez Dumm INT, Pezzano H. 1981. Early effects of EFA deficiency on the structure and enzymatic activity of rat liver microsomes. Prog Lipid Res 20: $315-321$.

24) Garg ML, Keelan M, Thomson ABR, Clandinin MT. 1992. Desaturation of linoleic acid in the small bowel is increased by short-term fasting and by dietary content of linoleic acid. Biochim Biophys Acta 1126: 17-25.

25) Salem N Jr, Reyzer M, Karanian J. 1996. Losses of arachidonic acid in rat liver after alcohol inhalation. Lipids 31: S153-S156.

26) Morimoto M, Reitz RC, Morin RJ, Nguyen K, Ingelman-Sundberg M, French SW. 1995. CYP-2E1 inhibitors partially ameliorate the changes in hepatic fatty acid composition induced in rats by chronic administration of ethanol and a high fat diet. J Nutr 125: 2953-2964.

27) Nanji AA, Sadrzadeh SMH, Dannenberg AJ. 1994. Liver microsomal fatty acid composition in ethanol-fed rats: Effect of different dietary fats and relationship to liver injury. Alcohol Clin Exp Res 18: 1024-1028.

28) Armstrong MK, Blake WL, Clarke SD. 1991. Arachidonic acid suppression of fatty acid synthase gene expression in cultured rat hepatocytes. Biochem Biophys Res Commun 177: 1056-1061.

29) Mann NJ, Warrick GE, O'Dea K, Howard HR, Sinclair AJ. 1994. The effect of linoleic, arachidonic and eicosapentaenoic acid supplementation on prostacyclin production in rats. Lipids 29: 157-162.

30) Nanji AA, Zakim D, Rahemitulla A, Daly T, Miao L, Zhao S, Khwaja S, Tahan SR, Dannenberg AJ. 1997. Dietary saturated fatty acids down-regulate cyclooxygenase-2 and tumor necrosis factor alfa and reverse fibrosis in alcohol-induced liver disease in the rat. Hepatology 26: 1538-1545.

31) Chedid A, Mendenhall CL, Moritz TE, French SM, Chen TS, Morgan TR, Roselle GA, Nemchausky BA, Tamburro CH, Schiff ER, McClain CJ, Marsano LS, Allen JI, Samanta A, Weesner RE, Henderson WG, Veterans Affairs Cooperative Study Group. 1993. Cell-mediated hepatic injury in alcoholic liver disease. Gastroenterology 105: 254-266.

32) Bursch W, Taper HS, Somer MP, Meyer S, Putz B, Schulte-Hermann R. 1989. Histochemical and biochemical studies on the effect of the prostacyclin derivative Iloprost on $\mathrm{CCl}_{4}$-induced lipid peroxidation in rat liver and its significance for hepatoprotection. Hepatology 9: 830-838.

33) Porta EA. 1997. Dietary modulation of oxidative stress in alcoholic liver disease in rats. J Nutr 127: 912S-915S. 